\title{
Neuroendocrine Tumors of the Gastrointestinal Tract
}

\author{
Chair: $\quad$ Andrea Frilling ${ }^{a}$ \\ Participants: Ken Herrmann ${ }^{\text {b }}$ Daniel Kämmerer ${ }^{c}$ Christos Toumpanakis ${ }^{d}$ \\ ${ }^{a}$ Department of Surgery and Cancer, Imperial College London, Hammersmith Campus, London, UK; \\ ${ }^{b}$ Department of Nuclear Medicine, University Hospital Essen, Essen, Germany; \\ ${ }^{c}$ Department of General and Visceral Surgery, Zentralklinik Bad Berka, Bad Berka, Germany; \\ ${ }^{d}$ Centre for GastroenterologyI Neuroendocrine Tumour Unit, ENETS Centre of Excellence, Royal Free Hospital, London, UK
}

\begin{abstract}
Question 1: Which imaging procedures do you consider effective in the diagnostic workup of patients with gastroenteropancreatic neuroendocrine tumors (GEP-NETs)? Should all patients with advanced neuroendocrine disease have both somatostatin receptor(SSR)-based imaging and ${ }^{18}$ F-fluorodeoxyglucose positron emission tomography/computed tomography ( ${ }^{18}$ F-FDG PET/CT)?
\end{abstract}

Herrmann: The appropriate image modality highly depends on the tumor grade. According to the most recent ENETS guidelines (2016), ${ }^{18} \mathrm{~F}-\mathrm{FDG}$ PET/CT is only recommended in G3 tumors. Whereas for G1 and G2 tumors, SSR-binding PET ligands (SSR PET; e.g. ${ }^{68} \mathrm{Ga}$-DOTATATE or ${ }^{68} \mathrm{Ga}$-DOTATOC) are recommended. However, there is some data investigating the combination of both imaging modalities. It has been shown that FDG positivity is associated with an inferior prognosis. Despite promising results suggesting the additional value of ${ }^{18} \mathrm{~F}$-FDG PET/CT in G2 tumors, the corresponding conclusions are still under debate. At our center, we do not routinely perform ${ }^{18} \mathrm{~F}-\mathrm{FDG}$ and SSR PET/CT in all G1 and G2 patients, but limit the additional use of ${ }^{18} \mathrm{~F}-\mathrm{FDG}$ to specific clinical scenarios. The most common indications include the identification of a potentially dedifferentiated lesion, biopsy guidance in a patient scheduled for re-biopsy, and for restaging in patients with morphologically growing but discordant SSR $\mathrm{PET} / \mathrm{CT}$ findings. In addition, the morphologic information provided by contrast-enhanced CT or magnetic resonance imaging (MRI) is very helpful. In initially diagnosed, well-differentiated GEP-NETs we often perform a 'one-stop shop' SSR PET/MRI, taking advantage of the superior detection rate of liver metastases by means of MRI.

Kämmerer: If a patient is referred for staging after first diagnosis, then I like to recommend a biopsy first in order to assess the proliferation index (Ki-67). Patients with G1 and G2 neuroendocrine neoplasms (NEN) with a proliferation index lower than $10 \%$ should receive a DOTATOC or DOTATATE PET/CT only. Cases of G2 NEN with a Ki-67 of more than $10 \%$ (but less than $20 \%$ ) proliferation rate, and/or with aggressive clinical progression should be imaged with both.

In patients with a G3 NEN (proliferation index > 20\%), an ${ }^{18} \mathrm{~F}$ FDG PET/CT is recommended at first. In cases of a mismatch of metabolic/receptor and morphological imaging, dual imaging should be considered.

Toumpanakis: In terms of cross-sectional imaging, I would always start with a good quality triple-phase CT of the chest, abdomen and pelvis with intravenous contrast in order to have an initial idea of the disease bulk/extent. If further information is needed with regards to the location of primary (primaries) in small-bowel NETs, a CT/MRI enterography can be considered. If hepatic surgery is considered, I would always request an MRI of the liver for better assessment of hepatic tumor load prior to surgical intervention. Finally, for better assessment of skeletal metastases, especially in the spine, an MRI spine seems to be a very accurate imaging modality.

Regarding molecular imaging, I am feeling that we are moving towards the replacement of somatostatin receptor scintigraphy (SRS) with ${ }^{111}$ In (OCTREOSCAN) by the new PETs, with ${ }^{68} \mathrm{Ga}$ DOTATOC (-TATE), as they have been proven to be more specific and more sensitive. However, as the latter have not been widely available, I still feel that OCTREOSCAN needs to be performed in the first place and if: a) OCTREOSCAN is negative, b) small-volume disease or bone metastases are suspected, and c) major interventional procedures are considered (such as hepatectomies or liver transplantation), then the patient will need to be referred for ${ }^{68} \mathrm{Ga}$-DOTATOC (-TATE) PET. The latter imaging studies are considered to be the most sensitive imaging modality in well-dif-

\section{KARGER}

(๑) 2017 S. Karger GmbH, Freiburg

Fax +497614520714
Prof. Andrea Frilling, MD, PhD, FACS, FRCS, FEBS 
ferentiated GEP-NETs. For localization of benign insulinomas that lack type 2 \& 5 SSR, glucagon-like peptide-1 receptor imaging will probably represent the imaging modality of choice in the future, whenever it becomes widely available. In those tumors, cross-sectional imaging and even ${ }^{68} \mathrm{Ga}$-DOTATOC (-TATE) PET quite often fail to localize the small pancreatic insulinoma. ${ }^{18} \mathrm{~F}$-FDG PET was initially considered as a useful molecular imaging modality only in high-grade GEP-NETs. In those tumors, it does indeed represent the imaging modality of first choice. However, its role has recently become more helpful even in grade 1/grade 2 tumors, which have been characterized as such on the basis of a 'single biopsy'. As GEP-NETs are highly heterogeneous neoplasms, ${ }^{18} \mathrm{~F}$ FDG PET may reveal tumor lesions of higher grade within the tumor bulk and justify the need for a guided biopsy of those FDGavid lesions, which will have implications on the choice of treatment and overall disease prognosis. Also, ${ }^{18} \mathrm{~F}$-FDG PET can reveal 'second primaries' such as lung adenocarcinomas that may co-exist with GEP-NETs. However, despite its increasing efficacy in all GEP-NETs, we would need more prospective studies before we suggest the concomitant use of SRS and ${ }^{18} \mathrm{~F}$-FDG PET in all patients with advanced GEP-NETs.

Finally, it is very important for all imaging modalities to be reviewed and interpreted side by side by experienced radiologists and nuclear medicine physicians in the NET multidisciplinary/tumor board meeting. On the one hand, molecular imaging modalities and especially PET imaging can reveal unexpected tumor lesions, which can then be retrospectively demonstrated in cross-sectional imaging, but on the other hand 'false-positive' lesions, such as ${ }^{68} \mathrm{Ga}$ PET physiological uptake in the uncinate process of the pancreas, need to be taken into account.

\section{Question 2: Do you consider tumor markers helpful for diagnosis of a NET and monitoring of treatment response, and which of them do you use in your practice? What should an ideal tumor marker deliver?}

Hermann: As nuclear medicine physicians we are only partially involved in the treatment of NETs. With peptide receptor radionuclide therapy (PRRT), however, we have a very potent treatment available in the post-NETTER-1 era. As not all patients respond to PRRT, it would be desirable to have a predictive biomarker/tumor marker at hand. The recent introduction of blood transcript analysis (NETest) seems to offer a promising, although not yet fully validated tool for identifying patients likely to benefit from PRRT.

Kämmerer: An ideal tumor marker is characterized by a high sensitivity and high disease specificity. Biomarkers should predict recurrences before they are clinically detectable. Additionally, the test should be cost-effective, safe, and independent of medical drugs. For NEN, the minimally required biochemical tests include plasma chromogranin A (CgA) and urinary 24-h 5-hydroxyindoleacetic acid (5-HIAA) for small-intestine neoplasms. Pancreatic neuroendocrine neoplasms (P-NEN) often demonstrate a lower specificity, particularly insulinoma. For sporadic P-NEN, serum CgA level can be used for follow-up. For functional P-NEN, it is recommended that biomarker diagnostics include the biologically active peptides that are secreted by the tumor cells (e.g. insulin, gastrin, and glucagon).

Toumpanakis: For diagnosis of a suspected functioning GEPNET, I always use the relevant peptide (fasting levels for ZollingerEllison syndrome, 24-h urine or plasma 5-HIAA for carcinoid syndrome, etc.), whilst I am also cautious in the interpretation of those results. For example, for establishing a diagnosis of gastrinoma apart from hypergastrinemia -, a gastric $\mathrm{pH}<2$ is also needed. I would not rely on CgA for a diagnosis of a non-functioning GEPNET, as it is quite often normal, especially in small-volume disease, whilst it can be raised in many non-NET clinical entities. For detection of possible carcinoid heart disease in patients with carcinoid syndrome, I would request the measurement of plasma $\mathrm{N}$ terminal pro B-type natriuretic peptide, as its role has been established in prospective studies.

For the monitoring of treatment response, I would still use the relevant peptides (as mentioned above) in patients with functioning GEP-NETs. I still routinely use CgA levels during the patients' follow-up; however, I do not make decisions based only on its levels. I feel that CgA still has a complementary role, especially in advanced midgut NETs. According to our experience and some good quality retrospective data, rising $\mathrm{CgA}$ levels in that group of patients may predict a forthcoming radiological progression. I would be really lucky though if I had some of the new tumor markers available in clinical practice. For example, circulating tumor cells seem to provide information about treatment response and disease prognosis, and also NETest may reveal early disease recurrence (even before the sensitive molecular imaging studies) and also seems to predict the response of several treatments.

The ideal tumor marker would provide, at the time of diagnosis, information about tumor recurrence after surgery, current tumor load, tumor behavior, response to certain treatments, and also the patient's overall survival.

\section{Question 3: A steadily increasing number of patients are diagnosed with small $(<2 \mathrm{~cm}$ in size), non-secreting NETs of the pancreas. How do you manage these patients? Are there any features on imaging, biochemistry, or endoscopic ultrasound (EUS) which could help to delineate the long-term biological behavior of these small lesions?}

Herrmann: Lesions with a diameter of $5 \mathrm{~mm}$ and greater can usually be well characterized by PET imaging. Especially well-differentiated NETs should be detectable on an SSR PET/CT.

Kämmerer: Firstly, the patients should receive an EUS and a biopsy to characterize the tumor (CgA, Syn, Ki-67). We also perform 
$\mathrm{a}^{68} \mathrm{Ga}$-DOTATATE PET/CT to exclude metastasis. If the tumor is located in the pancreas corpus/tail, we try to remove the tumor laparoscopically because tumors larger than $1.5 \mathrm{~cm}$ can metastasize into lymph nodes quite early (the ENETS guidelines advise resection by more than $2 \mathrm{~cm}$ ). If the tumor is located in the pancreas head, we will discuss the findings with the patients in detail. NEN localized in the pancreas head $(>1.5 \mathrm{~cm})$ should be removed surgically. Today, pancreatic head resection has become an extremely safe operation in specialized departments. A functional small tumor (e.g. benign insulinoma) can be treated by enucleation. Biomarkers to predict clinical behavior are not known.

Toumpanakis: First, I take into account the patients' performance status and other comorbidities. Second, I discuss the case in our NET multidisciplinary/tumor board meeting and clarify with my hepato-pancreato-biliary surgical colleagues the type of operation they are considering for that specific tumor. If, for example, there is a $1.5 \mathrm{~cm}$ tumor in the tail of the pancreas, which can be managed with a distal pancreatectomy in a patient with no significant comorbidities, I would probably refer the patient for surgery. On the contrary, I would not refer in the first instance an elderly patient with a sporadic/incidentally diagnosed $(<2 \mathrm{~cm}) /$ non-functioning pancreatic head NET for surgery, as the risks of a pancreatoduodenectomy in that particular patient outweigh the benefits. In that scenario, I would consider active surveillance or commencement of lanreotide Autogel (if the tumor is avid on SRS) for control of tumor growth, on the basis of CLARINET data. We are looking forward of course to hearing the results of the new prospective trial, under ENETS' auspices, that will investigate the long-term behavior of those $<2 \mathrm{~cm}$, non-functioning pancreatic NETs (p NETs).

Unfortunately, to date, we do not seem to have a reliable biomarker available that can predict the behavior of a small p NET. In terms of imaging, if the tumor shows avidity in SRS, this may indicate, in combination with EUS cytology/histology, that it is a welldifferentiated tumor. On the contrary, ${ }^{18} \mathrm{~F}$-FDG PET avidity, even if cytology/histology indicate a well-differentiated tumor, may predict a more aggressive 'behavior'. In those cases, I would refer for surgery earlier, especially if there are no significant comorbidities, or I would commence the patient on medical treatment to control tumor growth. Therefore, in cases where there are some concerns about the long-term behavior of the small p NET, I would arrange both a ${ }^{68} \mathrm{Ga}$ PET and an FDG PET.

\section{Question 4: A large proportion of patients with GEP-NETs presents in advanced tumor stage. Which criteria do you use for selection of treatment? Is tumor downstaging a realistic approach and which treatment options would you choose?}

Herrmann: Using PRRT for presurgical, neoadjuvant downstaging has been widely discussed but only very limited data is available so far. However, the Rotterdam group reported promising results in 29 patients undergoing neoadjuvant PRRT. These results warrant further prospective studies.

Kämmerer: Most of the patients are metastasized at the stage of diagnosis of the disease. Most of them present liver and lymph node metastasis. We like to perform a complete morphologic and receptor status $\left({ }^{68} \mathrm{Ga}\right.$-DOTATOC or DOTATATE PET/CT; \pm MRI liver, endosonography). For G3 NEN, we like to perform an ${ }^{18} \mathrm{~F}$ FDG PET/CT as well.

If the primary tumor is resectable, we would remove it in G1/G2 tumors, even in the metastasized stage. We try to downsize advanced or borderline resectable G1/G2 primary tumors.

If the G1/G2 neoplasms demonstrate a high standardized uptake value (SUV) (= high SSR status) in ${ }^{68} \mathrm{Ga}$-SSTR PET, we like to perform a PRRT with Lu-177 to downsize the tumor burden. There is an exception: If a desmoplastic reaction is visible in CT scan, we recommend to remove as early as possible to avoid intestine ischemia in patients with small-bowel neoplasms.

For G3 carcinoma, chemotherapy treatment would be our first choice of therapy. If the follow-up diagnostic present a response, we will then continue the treatment with a following surgical approach.

Toumpanakis: For selection of treatment in patients with advanced GEP-NETs, I utilize the following criteria: patient performance status and comorbidities, patient symptoms, tumor differentiation and grade, tumor load, tumor primary site, tumor status (stable or progressing), avidity on SRS or on ${ }^{18} \mathrm{~F}-\mathrm{FDG}$ PET, presence and severity of carcinoid heart disease and mesenteric fibrosis. Finally, I always take into account the patient's preferences and quality of life issues. Ideally, I would also like to have some predictive biomarkers for each treatment option, but this is not possible at present. Finally, I discuss the patient case with my colleagues in the NET multidisciplinary/tumor board meeting to ensure that all of the above factors are taken into account and that the treatment is individualized for every single patient.

For patients who do not have significant comorbidities, if the tumor lesions are borderline resectable in the pancreas or in the liver, we can consider downstaging treatment. In our institution, we utilize either systemic chemotherapy (in p NETs especially with Ki-67 $>10 \%$ ) or transcatheter arterial embolization of hepatic metastases. Following the completion of those treatments, which can be associated with a radiological objective response in $30-40 \%$ of patients, we re-discuss the cases in the NET multidisciplinary/ tumor board meeting and refer the patients to hepato-pancreatobiliary surgeons if appropriate. So far, we have not used PRRT treatment for that indication as we are waiting for more data.

Question 5: What is the role of transplantation in the treatment of patients with NETs? How should a transplant candidate be investigated? Should liver transplantation be considered at an early stage of a treatment journey? 
Kämmerer: Liver transplantation in NET patients is and should be really rare. With regard to the available organs in Europe we have to be careful to select suitable patients with a good outcome. Also, the split-liver procedure has to be kept in mind but we have to acknowledge that there is still a risk for the donor. A suitable patient is characterized by a tumor with a Ki-67 lower than $5 \%$ (primary tumor and liver metastasis) and a younger age. The primary tumor has to be removed prior to transplantation. Extrahepatic tumor spread metastases have to be excluded by a ${ }^{68} \mathrm{Ga}-\mathrm{DO}$ TATOC or DOTATATE PET/CT every 6 months while the patient is on the waiting list. The liver metastases should be treated by a bridging therapy in between (e.g. PRRT, if there is a high SUV in the PET scan, and/or interventional radiological embolism due to a lower SUV). Apart from the concomitant liver treatment, the patient should be in good condition without any major comorbidities. Based on many available local and systemic treatment options in patients with low proliferative tumors (G1-G2), liver transplantation should be a last resort due to the limitation of available organs in Europe.

Toumpanakis: There is a role for liver transplantation in patients with GEP-NET, but in highly selected cases. In our institution, we can consider a patient for transplantation if: age $<60$, tumor grade is G1, the disease has been radiologically stable for at least 3 years, there is evidence of poorly controlled hormonal syndrome, there is no evidence of carcinoid heart disease, there is no evidence of extrahepatic disease, and following thorough assessment.

In terms of the latter, we arrange in all transplantation candidates a ${ }^{68} \mathrm{Ga}$ PET and an ${ }^{18} \mathrm{~F}$-FDG PET to ensure that there is no evidence of extrahepatic disease. Then we discuss those images alongside with the cross-sectional imaging in the NET multidisciplinary/tumor board meeting. Lesions with discrepancies between cross-sectional and molecular imaging are biopsied.

I am not sure that liver transplantation should be considered earlier, as in those cases, we may note earlier recurrences of disease. I feel that we should still be highly selective, follow strict criteria, and make sure that there is clearly no evidence of extrahepatic disease.

\section{Participants}

Prof. Dr. med. Ken Herrmann

Klinik für Nuklearmedizin

Universitätsklinikum Essen

Hufelandstraße 55, 45147 Essen, Germany

Ken.Herrmann@uk-essen.de

PD Dr. med. Daniel Kämmerer

Klinik für Allgemeine Chirurgie/Viszeralchirurgie

Zentralklinik Bad Berka

Robert-Koch-Allee 9

99437 Bad Berka, Germany

Daniel.Kaemmerer@zentralklinik.de

Dr. Christos Toumpanakis, MD PhD FRCP

Centre for Gastroenterology|Neuroendocrine Tumour Unit ENETS Centre of Excellence, Royal Free Hospital

Pond Street, London NW3 2QG, UK

c.toumpanakis@ucl.ac.uk

www.royalfree.nhs.uk/net 